\title{
CONTROLE DE SOJA VOLUNTÁRIA COM HERBICIDAS REGISTRADOS PARA ALGODOEIRO
}

\section{CONTROL OF VOLUNTARY SOYBEAN PLANTS WITH HERBICIDES RECOMMENDED FOR COTTON}

Giovana Oliveira Takahashi ${ }^{\mathrm{a}}$, Guilherme Braga Pereira Braz ${ }^{\mathrm{a} *}$, Fellipe Goulart Machado ${ }^{\mathrm{b}}$, Alberto Leão de Lemos Barroso ${ }^{\mathrm{a}}$, Antônio Jussiê da Silva Solino ${ }^{\mathrm{c}}$

${ }^{\mathrm{a}}$ Faculdade de Agronomia, Universidade de Rio Verde, Goiás, Brasil. ${ }^{\mathrm{b}}$ Universidade Estadual de Maringá, Paraná, Brasil. ${ }^{\mathrm{C}}$ De Lollo Agronegócios, Goiás, Brasil.

*Autor correspondente: guilhermebrag@gmail.com.

\section{INFORMAÇÕES DO ARTIGO}

\section{Histórico do artigo:}

Recebido: 09 Março 2020.

Aceito: 17 Março 2021.

Publicado: 31 Março 2021

\section{Palavras-chave/Keywords:}

Glycine max/ Glycine max.

Gossypium hirsutum/ Gossypium

hirsutum.

Herbicidas em pós-emergência/ Postemergence herbicides.

\section{Financiamento: \\ Universidade de Rio Verde - UniRV.}

Direito Autoral: Este é um artigo de acesso aberto distribuído sob os termos da Licença Creative Commons, que permite uso, distribuição e reprodução irrestritos em qualquer meio, desde que $\mathrm{o}$ autor e a fonte originais sejam creditados

\section{Citação deste artigo:}

TAKAHASHI, G. O.; BRAZ, G. B. P.; MACHADO, F. G.; BARROSO, A. L. L.; SOLINO, A. J. S. Controle de soja voluntária com herbicidas registrados para algodoeiro. Revista Brasileira de Herbicidas, v. 19, n. 4. 2020.

\begin{abstract}
RESUMO
Em parte da região Centro-Oeste do Brasil o algodoeiro tem sido cultivado em segunda safra, semeado após a colheita de soja. Nestas áreas, tem sido comum a ocorrência de plantas voluntárias de soja interferindo no desenvolvimento do algodoeiro. Neste sentido, o objetivo deste trabalho foi avaliar a eficácia de herbicidas aplicados em pós-emergência no algodoeiro para o controle de soja voluntária contendo diferentes transgenias. Dois experimentos foram conduzidos em casa de vegetação, utilizando um cultivar de soja com tecnologia Liberty Link ${ }^{\circledR}\left(\mathrm{LL}^{\circledR}\right)$, que confere tolerância ao glufosinate, e no outro cultivar Roundup Ready ${ }^{\circledR}\left(R^{\circledR}\right)$, que possui tolerância ao glyphosate. Em ambos os experimentos foi utilizado o delineamento de blocos casualizados, em arranjo fatorial $7 \times 2$, com 4 repetições. O primeiro fator foi constituído por herbicidas registrados para o algodoeiro: 2,4-D, dicamba, glyphosate, pyrithiobac, trifloxysulfuron, S-metolachlor, além de uma testemunha sem aplicação; enquanto que o segundo fator consistiu da associação ou não com o glufosinate. Para aferir o desempenho dos tratamentos herbicidas, foram realizadas avaliações de porcentagem de controle, altura e massa seca de parte aérea das plantas de soja. Para soja voluntária $L L^{\circledR}$, dicamba e glyphosate isolados, além da associação de glufosinate com dicamba, 2,4-D ou glyphosate, consistiram nos tratamentos com maior eficácia, proporcionando níveis de controle acima de $80,0 \%$. Para a soja $R^{\circledR}{ }^{\circledR}$, dicamba, trifloxysulfuron e 2,4-D isolados apresentaram eficácia no controle das plantas voluntárias, visualizando-se incrementos nos níveis de controle quando se procedeu a adição de glufosinate à calda de aplicação destes herbicidas.
\end{abstract}

\begin{abstract}
In part of the Midwest region of Brazil, cotton has been grown in second crop, sown after soybean harvest. In these areas, the occurrence of voluntary soybean plants has been common, interfering in the development of cotton crop. In this sense, the objective of this work was to evaluate the effectiveness of herbicides applied post-emergence in cotton for the control of voluntary soybeans containing different transgenics. Two experiments were carried out in a greenhouse, using a soybean cultivar with Liberty Link ${ }^{\circledR}$ technology $\left(\operatorname{LL}^{\circledR}\right)$, which confers tolerance to glufosinate, and the other Roundup Ready ${ }^{\circledR}\left(\operatorname{RR}^{\circledR}\right)$ cultivar, which has tolerance to glyphosate. In both experiments, a randomized completely block design was used, in a $7 \times 2$ factorial arrangement, with 4 replications. The first factor consisted of herbicides registered for cotton: 2,4-D, dicamba, glyphosate, pyrithiobac, trifloxysulfuron, S-metolachlor, in addition to a control without application; while the second factor consisted of the association or not with glufosinate. To assess the performance of herbicide treatments, evaluations were made of the percentage of control, height and shoot dry mass of soybean plants. For LL ${ }^{\circledR}$ voluntary soybeans, dicamba and glyphosate alone, in addition to the association of glufosinate with dicamba, 2,4-D or glyphosate, they consisted of the most effective treatments, providing control levels above $80.0 \%$. For RR ${ }^{\circledR}$ soybeans, dicamba, trifloxysulfuron and 2,4-D isolated showed efficacy in the control of voluntary plants, with increases in control levels being seen when the addition of glufosinate was applied to the spray solution of these herbicides.
\end{abstract}


G. O. TAKAHASHI et al.

\section{Introdução}

O algodão, matéria prima no setor têxtil, representa uma das principais commodities agrícolas a nível global. $\mathrm{O}$ Brasil encontra-se entre os cinco maiores produtores mundiais de algodoeiro (USDA, 2021), apresentando potencial de aumento na produção de forma qualitativa e quantitativa. Nas duas últimas safras, visualizou-se aumento significativo na área de cultivo de algodoeiro, principalmente pelos grandes investimentos no setor $\mathrm{e}$ expansão na área cultivada nos Estados do Mato Grosso e Bahia, que correspondem a aproximadamente a $88 \%$ da estimativa de produção na safra de 2019/2020 (CONAB, 2020).

Atualmente na região Centro-Oeste do Brasil, onde se localiza a maior parte do bioma Cerrado, o algodoeiro herbáceo tem sido tradicionalmente semeado de novembro a dezembro e colhido de junho a julho, em condições de cultivo que se caracterizam como de primeira safra. No entanto, o cultivo do algodoeiro em segunda safra ("safrinha"), seguido da colheita de soja precoce tem ganhado espaço entre os produtores, uma vez que possibilita menor uso de insumos e maior rentabilidade, se comparado ao cultivo nesta época com outras culturas agrícolas (BRAZ et al., 2013a).

Apesar dos benefícios do cultivo de segunda safra, neste sistema, plantas voluntárias de soja devem ser controladas durante o desenvolvimento do algodoeiro, uma vez, que a soja pode emergir a partir de grãos que foram debulhados das vagens antes da colheita (BOND; WALKER, 2009), ou daqueles que apresentaram anomalias na germinação, e ainda, de grãos oriundos de perdas durante a colheita (TOLEDO et al., 2008). O rápido crescimento das plantas voluntárias de soja causa sombreamento no algodoeiro, o qual apresenta desenvolvimento inicial lento, fato que ocasiona redução da taxa fotossintética e influencia negativamente a cultura (CARDOSO et al., 2010; BRAZ et al., 2013a; FERREIRA et al., 2015).

Os danos da interferência das plantas voluntárias de soja no algodoeiro ocorrem principalmente no desenvolvimento inicial da cultura, uma vez que a soja voluntária é considerada uma planta daninha de difícil controle devido à escassez de herbicidas com espectro de controle sobre folhas largas disponíveis para o algodoeiro (LEE et al., 2009). Essas opções são ainda menores, com o advento de novas tecnologias que conferem tolerância à herbicidas para as cultivares de soja, impossibilitando o uso de determinados herbicidas que apresentavam eficácia no controle destas plantas voluntárias (GREEN, 2009). Assim, a escolha da cultivar a ser semeada antes do algodoeiro torna-se uma das principais estratégias para evitar o surgimento da soja voluntária nos cultivos de algodão em segunda safra.

Entre as alternativas de latifolicidas registradas para aplicações em pós-emergência de cultivares de algodoeiro convencional, estão pyrithiobac-sodium e trifloxysulfuron- sodium, ambos possuindo como mecanismo de ação a inibição da enzima acetolactato sintase (ALS). Para cultivares de algodoeiro transgênico com as tecnologias Roundup Ready ${ }^{\circledR}$, tolerante ao glyphosate $\left(\mathrm{RR}^{\circledR}\right)$ e Liberty Link $^{\circledR}$, tolerante ao glufosinate $\left(\mathrm{LL}^{\circledR}\right)$, há possibilidade de se aplicar em pós-emergência das plantas os herbicidas glyphosate e glufosinate, respectivamente. Além destes, outro produto que pode ser aplicado após a emergência das plântulas de algodoeiro é o S-metolachlor, que apresenta registro para aplicação quando a cultura se encontra em estádio popularmente conhecido por "orelha de onça", que se caracteriza pela presença apenas das folhas cotiledonares (over the top) (OLIVEIRA NETO et al., 2015).

Por fim, nos próximos anos haverá entrada no mercado de cultivares de algodoeiro nas quais será possível a utilização de maneira seletiva dos herbicidas dicamba e 2,4-D. A partir deste contexto, fica evidente a necessidade de se realizar estudos que visem identificar alternativas de herbicidas que possam ser empregados para o controle de plantas voluntárias de soja na cultura do algodoeiro. Diante deste contexto, o objetivo do presente trabalho foi avaliar a eficácia de herbicidas aplicados em pós-emergência do algodoeiro no controle de plantas voluntárias de soja.

\section{Material e Métodos}

Os experimentos foram realizados em casa de vegetação no campus sede da Universidade de Rio Verde, em Rio Verde (GO), de 07 de janeiro de 2019 a 08 de março de 2019. Em cada experimento foi utilizada um cultivar de soja distinta, as quais diferiram em função da tecnologia que apresentam para tolerância à herbicidas. Em um dos experimentos foi utilizada a cultivar de soja CZ37B19 LL ${ }^{\circledR}$, material tolerante ao herbicida glufosinate e no outro a cultivar BRS Flecha RR2 IPRO ${ }^{\circledR}$, que apresenta tolerância ao glyphosate.

Em ambos os experimentos, as unidades experimentais foram compostas por um vaso com capacidade de $2 \mathrm{dm}^{3}$ de solo. $O$ solo utilizado no preenchimento dos vasos foi coletado em área experimental em que o mesmo foi classificado como Latossolo Vermelho distroférrico (EMBRAPA, 2018). A análise laboratorial do solo apresentou as seguintes propriedades físico-químicas: $\mathrm{pH} \mathrm{CaCl} 2=6,2 ; \mathrm{P}=89,01 \mathrm{mg} \mathrm{dm}{ }^{-3} ; \mathrm{Ca}^{2+}=5,75, \mathrm{Mg}^{2+}=$ 2,$35 ; \mathrm{K}^{+}=0,51 ; \mathrm{Al}^{3+}=0,00 ; \mathrm{H}+\mathrm{Al}=1,43 ;$ M.O. $=$ $50,5 \mathrm{~g} \mathrm{dm}^{-3}$; areia $=63 \%$, silte $=12 \%$ e argila $=25 \%$ (textura franco argilo-arenosa).

O delineamento experimental utilizado nos dois experimentos foi o de blocos completos ao acaso, em arranjo fatorial $7 \times 2$, com 4 repetições. $\mathrm{O}$ primeiro fator correspondeu a diferentes herbicidas posicionados para o algodoeiro em aplicações em pós-emergência da cultura, além de uma testemunha sem herbicida; enquanto que o segundo fator consistiu na aplicação em associação ou não com o glufosinate (Tabela 1). 
G. O. TAKAHASHI et al.

Tabela 1. Relação de herbicidas avaliados no experimento visando ao controle em pós-emergência de plantas voluntárias de soja.

\begin{tabular}{|c|c|c|c|}
\hline Herbicida isolado & Associação & Dose $\left(\mathrm{g} \mathrm{ha}^{-1}\right)$ & Produtos comerciais \\
\hline 2,4-D amina & Com glufosinate & $644+200^{\frac{1}{I}}$ & DMA 806 BR $^{(\mathbb{R}}+$ Finale $^{(\mathbb{R}}$ \\
\hline 2,4-D amina & Sem glufosinate & 644 & DMA 806 BR $^{\circledR}$ \\
\hline Dicamba & Com glufosinate & $600+200^{1 /}$ & Atectra $^{\circledR}+$ Finale $^{\circledR}$ \\
\hline Dicamba & Sem glufosinate & 600 & Atectra ${ }^{\circledR}$ \\
\hline Glyphosate & Com glufosinate & $480+200^{1 /}$ & Roundup Original $^{\circledR}+$ Finale $^{\circledR}$ \\
\hline Glyphosate & Sem glufosinate & 480 & Roundup Original $^{(\mathbb{B})}$ \\
\hline Pyrithiobac-sodium & Com glufosinate & $84+200^{1 /}$ & Staple ${ }^{\mathbb{B}}+$ Finale $^{\mathbb{B}}$ \\
\hline Pyrithiobac-sodium & Sem glufosinate & $84^{\underline{1 /}}$ & Staple $^{(\mathbb{R})}$ \\
\hline Trifloxysulfuron-sodium & Com glufosinate & $3+200^{1 /}$ & Envoke $^{\circledR}+$ Finale $^{\circledR}$ \\
\hline Trifloxysulfuron-sodium & Sem glufosinate & $3^{1 /}$ & Envoke $^{(\mathbb{R}}$ \\
\hline S-metolachlor & Com glufosinate & $1152+200^{\frac{1 /}{-}}$ & Dual Gold ${ }^{\mathbb{R}}+$ Finale $^{\mathbb{R}}$ \\
\hline S-metolachlor & Sem glufosinate & $1152^{\frac{1}{\prime}}$ & Dual Gold $^{\mathbb{R}}$ \\
\hline Sem herbicida & Com glufosinate & $200^{\frac{1}{1}}$ & Finale $^{\circledR}$ \\
\hline Testemunha sem herbicida & & & - \\
\hline
\end{tabular}

$\underline{1 /}$ Adicionado adjuvante Iharol Gold ${ }^{\circledR}$ na dose de $0,5 \% \mathrm{v} \mathrm{v}^{-1}$.

Na semeadura, foram distribuídas quatro sementes de soja por unidade experimental. Após a emergência das plântulas, foi realizado o raleio deixando apenas uma planta por vaso. Ao longo do período de condução do experimento, os vasos foram irrigados diariamente conforme necessidade hídrica das plantas de soja. As aplicações foram realizadas em pós-emergência das plantas de soja, quando estas encontravam-se no estádio V3/V4 (3 a 4 trifólios completamente expandidos). Para realizar a aplicação foi utilizado pulverizador costal a base de $\mathrm{CO}_{2}$, munido de três pontas XR110.02, mantido à pressão de trabalho de $240 \mathrm{kPa}$, resultando em volume de calda equivalente a $200 \mathrm{~L} \mathrm{ha}^{-1}$

Foi avaliada a porcentagem de controle da soja voluntária aos 7, 14, 21 e 28 dias após a aplicação (DAA) (SBCPD, 1995). Aos 28 DAA foram realizadas avaliações de altura e massa seca das plantas de soja. A avaliação de altura foi realizada com auxílio de régua, medindo a distância correspondente ao nível do solo até o meristema apical da planta de soja. Na avaliação de massa seca de parte aérea, o material foi coletado nos vasos e acondicionados em sacos de papel Kraft, os quais foram colocados em estufa de circulação forçada de ar pelo período de $72 \mathrm{~h}$ com temperatura constante de $65^{\circ} \mathrm{C}$.

Os dados foram processados no software estatístico SISVAR (FERREIRA, 2011) e submetidos à análise de variância após verificar a normalidade dos dados. Quando significativo ao teste $\mathrm{F}(p \leq 0,05)$, utilizou-se o teste de comparação de médias de Tukey $(p \leq 0,05)$.

\section{Resultados e Discussão}

\section{Desempenho sobre plantas voluntárias de soja com tolerância ao glufosinate $\left(\mathrm{LL}^{\circledR}\right)$}

A partir dos resultados obtidos nas avaliações da porcentagem de controle das plantas voluntárias de soja oriundas de cultivar com tolerância ao glufosinate (CZ37B19 LL $^{\circledR}$ ), foi possível determinar o desempenho dos tratamentos herbicidas aplicados em pós-emergência
(Tabela 2). A aplicação dos herbicidas dicamba e glyphosate, tanto de forma isolada quanto em associação com glufosinate, consistiu nos tratamentos com melhores performances de controle de soja voluntária aos 7 DAA, apresentando níveis variando entre 73,8 e $80,0 \%$.

Aos 14 DAA, os tratamentos que proporcionaram as maiores porcentagens de controle de soja voluntária foram compostos pelas associações de glufosinate com dicamba, 2,4-D ou glyphosate, além do dicamba aplicado isoladamente. Estes tratamentos foram semelhantes entre si e proporcionaram níveis de controle superiores à $90 \%$, fato que os credenciam como alternativas altamente eficazes sob as plantas voluntárias de soja já aos 14 DAA (Tabela 2). Devido ao sistema de cultivo adensado de segunda safra ser empregado nas áreas de produção, a velocidade de dessecação dos tratamentos é fator fundamental para manutenção da produtividade da cultura.

Conforme descrito por Raimondi et al. (2017), quando se realiza a semeadura adensada do algodão em segunda safra, o período anterior a interferência (PAI) é de apenas 11 dias após a emergência. $\mathrm{O}$ resultado apresentado na literatura indica que a soja voluntária necessita ser controlada precocemente no algodoeiro, uma vez que em menos de duas semanas após a emergência da cultura, esta apresenta-se sensível a interferência imposta pela comunidade infestante (plantas daninhas e voluntárias) com a qual convive. Como, o período de semeadura em segunda safra é limitado, programas de manejo que proporcionam alta velocidade de dessecação são essenciais para manutenção dos níveis de produtividade da cultura, destacando-se a possibilidade do uso de tratamentos que alcancem o patamar mínimo de $80,0 \%$ em um menor intervalo de tempo.

A partir da avaliação realizada aos 21 DAA, os tratamentos com os melhores desempenhos foram compostos por glyphosate e dicamba aplicados de forma isolada, além da associação de glufosinate aos herbicidas 2,4-D, glyphosate ou dicamba (Tabela 2). Além dos tratamentos supracitados, a aplicação de trifloxysulfuron + glufosinate foi capaz de impor níveis de controle acima de $80,0 \%$, credenciando esta associação como mais uma alternativa eficaz para o manejo químico de soja voluntária.

Rev. Bras. Herb., v. 19, n. 4, e. 707, out./dez., 2020 
G. O. TAKAHASHI et al.

Tabela 2. Porcentagem de controle de plantas voluntárias de soja, cultivar CZ37B19 LL ${ }^{\circledR}$ (tolerante ao glufosinate), submetidas à aplicação de herbicidas em pós-emergência. Rio Verde (GO), 2019.

\begin{tabular}{|c|c|c|c|c|}
\hline \multirow{2}{*}{ Herbicida $\left(\mathrm{g} \mathrm{ha}^{-1}\right)$} & \multicolumn{2}{|c|}{7 DAA } & \multicolumn{2}{|c|}{14 DAA } \\
\hline & Sem AG & Com $\mathrm{AG}^{1 /}$ & Sem AG & $\operatorname{Com} \mathrm{AG}^{1 /}$ \\
\hline 2,4-D (644) & $52,5 \mathrm{Bc}$ & $70,0 \mathrm{Ac}$ & $57,5 \mathrm{Bd}$ & $99,3 \mathrm{Aa}$ \\
\hline Dicamba (600) & $73,8 \mathrm{Aa}$ & $76,3 \mathrm{Aab}$ & $99,5 \mathrm{Aa}$ & $100,0 \mathrm{Aa}$ \\
\hline Glyphosate (480) & $78,8 \mathrm{Aa}$ & $80,0 \mathrm{Aa}$ & $91,0 \mathrm{Ab}$ & $94,5 \mathrm{Aa}$ \\
\hline Pyrithiobac (84) & $51,3 \mathrm{Bc}$ & $62,5 \mathrm{Ad}$ & $52,5 \mathrm{Bd}$ & $62,5 \mathrm{Ac}$ \\
\hline Trifloxysulfuron (3) & $61,3 \mathrm{Bb}$ & $73,8 \mathrm{Abc}$ & $68,3 \mathrm{Bc}$ & $77,0 \mathrm{Ab}$ \\
\hline S-metolachlor (1152) & $15,0 \mathrm{Ad}$ & $16,3 \mathrm{Ae}$ & 7,5 Ae & $10,0 \mathrm{Ad}$ \\
\hline Sem herbicida & $0,0 \mathrm{Ae}$ & $0,0 \mathrm{Af}$ & $0,0 \mathrm{Ae}$ & $0,0 \mathrm{Ae}$ \\
\hline \multirow[t]{3}{*}{ CV (\%) } & \multicolumn{2}{|c|}{5,53} & \multicolumn{2}{|c|}{6,56} \\
\hline & \multicolumn{2}{|c|}{$21 \mathrm{DAA}$} & \multicolumn{2}{|c|}{$28 \mathrm{DAA}$} \\
\hline & Sem AG & Com $\mathrm{AG}^{1 /}$ & Sem AG & Com $\mathrm{AG}^{1 /}$ \\
\hline $2,4-\mathrm{D}(644)$ & $64,5 \mathrm{Bb}$ & $100,0 \mathrm{Aa}$ & $57,5 \mathrm{Bc}$ & $100,0 \mathrm{Aa}$ \\
\hline Dicamba (600) & $99,0 \mathrm{Aa}$ & $100,0 \mathrm{Aa}$ & $98,8 \mathrm{Aa}$ & $100,0 \mathrm{Aa}$ \\
\hline Glyphosate (480) & $97,5 \mathrm{Aa}$ & $98,0 \mathrm{Aab}$ & $93,3 \mathrm{Aa}$ & $94,5 \mathrm{Aa}$ \\
\hline Pyrithiobac (84) & $63,8 \mathrm{Bb}$ & $75,0 \mathrm{Ac}$ & $50,0 \mathrm{Bc}$ & $56,3 \mathrm{Ac}$ \\
\hline Trifloxysulfuron (3) & $70,8 \mathrm{Bb}$ & $82,5 \mathrm{Abc}$ & $71,3 \mathrm{Bb}$ & $80,0 \mathrm{Ab}$ \\
\hline S-metolachlor (1152) & $22,5 \mathrm{Bc}$ & 42,5 Ad & $13,8 \mathrm{Ad}$ & $13,8 \mathrm{Ad}$ \\
\hline Sem herbicida & $0,0 \mathrm{Ad}$ & $0,0 \mathrm{Ae}$ & $0,0 \mathrm{Ae}$ & $0,0 \mathrm{Ae}$ \\
\hline CV (\%) & \multicolumn{2}{|c|}{11,69} & \multicolumn{2}{|c|}{7,03} \\
\hline
\end{tabular}

${ }^{1 /}$ Com $\mathrm{AG}=$ associado ao glufosinate $\left(200 \mathrm{~g} \mathrm{ha}^{-1}\right)$. * Médias seguidas por letras distintas maiúsculas na linha e minúsculas na coluna diferem entre si pelo teste de Tukey $(p \leq 0,05)$.

Apesar da cultivar de soja utilizada no presente experimento possuir tecnologia que confere tolerância ao glufosinate $\left(\mathrm{LL}^{\circledR}\right)$, visualizou-se para os tratamentos contendo pyrithiobac, trifloxysulfuron e 2,4-D melhorias na performance de controle quando foi realizada a associação destes ao glufosinate, em comparação com a aplicação destes herbicidas de forma isolada. Sobretudo, o referido comportamento foi mais evidente quando se utilizou o 2,4-D, uma vez que em aplicação isolada, os níveis de controle médios de soja voluntária nas quatro avaliações foram $\approx 34,3 \%$ inferiores em relação ao obtido pela associação entre 2,4-D e glufosinate.

Na literatura já foi relatado o efeito sinérgico da associação de 2,4-D + glufosinate, quando aplicada em pósemergência de espécies tolerantes e/ou resistente ao glyphosate (CRAIGMYLE; ELLIS; BRADLEY, 2013; BOTHA et al., 2014). Apesar disto, o fato que traz atenção especial no presente trabalho refere-se ao efeito sinérgico de controle ocorrer sob plantas geneticamente modificadas com tecnologia que confere tolerância ao glufosinate, criando a necessidade de estudos que possam elucidar o comportamento observado.

Além das avaliações de porcentagem de controle, aos 28 DAA, foram mensuradas a altura de plantas e massa seca de parte aérea, estando os resultados apresentados na Tabela 3. Para altura de plantas, menores valores desta variável-resposta foram observados nos tratamentos com aplicação de glyphosate isolado, além das associações de glufosinate com os herbicidas glyphosate, 2,4-D, dicamba ou trifloxysulfuron.

Tabela 3. Altura e massa seca de parte aérea de plantas voluntárias de soja, cultivar CZ37B19 LL ${ }^{\circledR}$ (tolerante ao glufosinate), submetidas à aplicação de herbicidas em pós-emergência. Rio Verde (GO), 2019.

\begin{tabular}{lcccc}
\hline \multirow{2}{*}{ Herbicida $\left(\mathrm{g} \mathrm{ha}^{-1}\right)$} & \multicolumn{2}{c}{ Altura de plantas $(\mathrm{cm})$} & \multicolumn{2}{c}{ Massa seca $(\mathrm{g})$} \\
\cline { 2 - 5 } & Sem AG & Com AG & Sem AG & ${\text { Com } \mathrm{AG}^{-1}}^{1 /}$ \\
\hline 2,4-D (644) & $29,3 \mathrm{Acd}$ & $31,5 \mathrm{Ac}$ & $3,4 \mathrm{Abc}$ & $1,6 \mathrm{Bc}$ \\
Dicamba (600) & $30,5 \mathrm{Acd}$ & $27,4 \mathrm{Ac}$ & $1,5 \mathrm{Ac}$ & $1,0 \mathrm{Ac}$ \\
Glyphosate (480) & $27,3 \mathrm{Ad}$ & $29,5 \mathrm{Ac}$ & $1,5 \mathrm{Ac}$ & $1,3 \mathrm{Ac}$ \\
Pyrithiobac (84) & $45,5 \mathrm{Ab}$ & $40,7 \mathrm{Ab}$ & $4,2 \mathrm{Aab}$ & $3,8 \mathrm{Aab}$ \\
Trifloxysulfuron (3) & $35,8 \mathrm{Ac}$ & $30,5 \mathrm{Ac}$ & $3,3 \mathrm{Abc}$ & $2,7 \mathrm{Abc}$ \\
S-metolachlor (1152) & $46,5 \mathrm{Ab}$ & $46,3 \mathrm{Ab}$ & $4,4 \mathrm{Aab}$ & $5,3 \mathrm{Aa}$ \\
Sem herbicida & $55,3 \mathrm{Aa}$ & $56,9 \mathrm{Aa}$ & $6,0 \mathrm{Aa}$ & $5,2 \mathrm{Aa}$ \\
\hline CV (\%) & \multicolumn{3}{c}{27,5} \\
\hline
\end{tabular}

1/ Com $\mathrm{AG}=$ associado ao glufosinate $\left(200 \mathrm{~g} \mathrm{ha}^{-1}\right) . *$ Médias seguidas por letras distintas maiúsculas na linha e minúsculas na coluna diferem entre si pelo teste de Tukey $(p \leq 0,05)$. 
G. O. TAKAHASHI et al.

De maneira análoga as avaliações de controle e altura, visualizou-se menores valores de massa seca de parte aérea das plantas voluntárias de soja com a aplicação dos herbicidas 2,4-D, dicamba, glyphosate e trifloxysulfuron isolados, e as associações destes com glufosinate. A redução percentual média de massa seca de parte aérea destes tratamentos, em comparação com à testemunha sem aplicação de herbicidas, foi de $\approx 66,0 \%$. Em relação à adição de glufosinate na composição dos tratamentos, verificou-se que apenas o 2,4-D apresentou diferenças na redução de massa seca quando se procedeu a associação entre herbicidas, proporcionando maior percentual de redução quando os ingredientes ativos (glufosinate $+2,4$-D) foram aplicados em associação do que de forma isolada (2,4-D).

\section{tolerância ao glyphosate $\left(R^{\circledR}{ }^{\circledR}\right)$}

Os resultados das quatro avaliações de controle de plantas voluntárias de soja tolerante ao glyphosate $\left(\mathrm{RR}^{\circledR}\right)$ submetidas à aplicação em pós-emergência de herbicidas estão apresentados na Tabela 4. Aos 7 DAA, os tratamentos que proporcionaram maiores níveis de controle sobre as plantas voluntárias de soja foram dicamba e trifloxysulfuron aplicados isoladamente, além das associações entre dicamba + glufosinate e 2,4-D + glufosinate. Ainda referente à primeira avaliação, excluindo-se o trifloxysulfuron, para todos os demais herbicidas, a adição de glufosinate à calda de aplicação proporcionou acréscimo nos níveis de controle de soja voluntária em comparação com a aplicação dos ingredientes ativos de forma isolada.

\section{Desempenho sobre plantas voluntárias de soja com}

Tabela 4. Porcentagem de controle de plantas voluntárias de soja, cultivar BRS Flecha RR2 IPRO ${ }^{\circledR}$ (tolerante ao glyphosate), submetidas à aplicação de herbicidas em pós-emergência. Rio Verde (GO), 2019.

\begin{tabular}{|c|c|c|c|c|}
\hline \multirow{2}{*}{ Herbicida $\left(\mathrm{g} \mathrm{ha}^{-1}\right)$} & \multicolumn{2}{|c|}{7 DAA } & \multicolumn{2}{|c|}{14 DAA } \\
\hline & Sem AG & Com $\mathrm{AG}^{1 /}$ & Sem AG & Com $\mathrm{AG}^{1 /}$ \\
\hline 2,4-D (644) & $61,3 \mathrm{Bb}$ & $76,3 \mathrm{Aab}$ & $53,0 \mathrm{Bd}$ & $99,5 \mathrm{Aa}$ \\
\hline Dicamba (600) & $69,5 \mathrm{Ba}$ & $78,8 \mathrm{Aa}$ & $100,0 \mathrm{Aa}$ & $100,0 \mathrm{Aa}$ \\
\hline Glyphosate (480) & $8,8 \mathrm{Bd}$ & 62,5 Ade & $8,8 \mathrm{Be}$ & $62,5 \mathrm{Ad}$ \\
\hline Pyrithiobac (84) & $60,0 \mathrm{Bb}$ & 66,3 Acd & $63,8 \mathrm{Bc}$ & $88,8 \mathrm{Ab}$ \\
\hline Trifloxysulfuron (3) & $68,8 \mathrm{Aa}$ & $71,3 \mathrm{Abc}$ & $77,5 \mathrm{Ab}$ & $80,5 \mathrm{Ac}$ \\
\hline S-metolachlor (1152) & $18,8 \mathrm{Bc}$ & 66,3 Acd & 7,5 Bef & 65,0 Ad \\
\hline Sem herbicida & $0,0 \mathrm{Be}$ & $58,8 \mathrm{Ae}$ & $0,0 \mathrm{Bf}$ & $68,8 \mathrm{Ad}$ \\
\hline \multirow[t]{3}{*}{ CV (\%) } & \multicolumn{2}{|c|}{4,59} & \multicolumn{2}{|c|}{5,80} \\
\hline & \multicolumn{2}{|c|}{$21 \mathrm{DAA}$} & \multicolumn{2}{|c|}{$28 \mathrm{DAA}$} \\
\hline & Sem AG & Com $\mathrm{AG}^{1 /}$ & Sem AG & Com $\mathrm{AG}^{1 /}$ \\
\hline $2,4-\mathrm{D}(644)$ & $84,3 \mathrm{Bbc}$ & $99,5 \mathrm{Aa}$ & $85,0 \mathrm{Bb}$ & $99,5 \mathrm{Aa}$ \\
\hline Dicamba (600) & $100,0 \mathrm{Aa}$ & $100,0 \mathrm{Aa}$ & $100,0 \mathrm{Aa}$ & $100,0 \mathrm{Aa}$ \\
\hline Glyphosate (480) & $35,0 \mathrm{Bd}$ & $62,3 \mathrm{Ac}$ & $7,5 \mathrm{Bc}$ & $70,0 \mathrm{Ab}$ \\
\hline Pyrithiobac (84) & $72,0 \mathrm{Bc}$ & $99,5 \mathrm{Aa}$ & $76,3 \mathrm{Bb}$ & $99,8 \mathrm{Aa}$ \\
\hline Trifloxysulfuron (3) & $96,3 \mathrm{Aab}$ & $98,0 \mathrm{Aab}$ & $88,8 \mathrm{Aab}$ & $97,0 \mathrm{Aa}$ \\
\hline S-metolachlor (1152) & $31,3 \mathrm{Bd}$ & $86,0 \mathrm{Ab}$ & $12,5 \mathrm{Bc}$ & $88,8 \mathrm{Aa}$ \\
\hline Sem herbicida & $0,0 \mathrm{Be}$ & $63,8 \mathrm{Ac}$ & $0,0 \mathrm{Bc}$ & $55,0 \mathrm{Ac}$ \\
\hline CV $(\%)$ & \multicolumn{2}{|c|}{8,27} & \multicolumn{2}{|c|}{8,62} \\
\hline
\end{tabular}

1/ Com $\mathrm{AG}=$ associado ao glufosinate $\left(200 \mathrm{~g} \mathrm{ha}^{-1}\right) . *$ Médias seguidas por letras distintas maiúsculas na linha e minúsculas na coluna diferem entre si pelo teste de Tukey $(p \leq 0,05)$.

Aos 14 DAA, os tratamentos com maiores percentuais de controle de soja voluntária foram os compostos pela aplicação isolada de dicamba, além das associações entre 2,4-D ou dicamba com glufosinate. Nos tratamentos supracitados, os níveis ficaram próximos de 100,0\%, indicando a elevada eficácia destes herbicidas em relação ao controle das plantas voluntárias de soja. Os tratamentos compostos pela aplicação de pyrithiobac ou trifloxysulfuron associado ao glufosinate também apresentaram eficácia em níveis satisfatórios $(\geq 80,0 \%)$ no controle de soja voluntária, contudo, o desempenho observado foi ligeiramente inferior ao obtido com a aplicação das associações entre glufosinate e mimetizadores da auxina (2,4-D e dicamba) ou do dicamba isolado.

Herbicidas pertencentes do grupo mimetizadores da auxina apresentam bom desempenho no controle de soja voluntária, sendo utilizados em larga escala para eliminação da soja antes do período de vazio sanitário (LIMA et al., 2011). Apesar disto, está previsto a partir da safra 2021/2022 a introdução de cultivares de soja transgênicas com tolerância ao 2,4-D e dicamba $\left(\right.$ Enlist $^{\circledR}$ e Intacta2 $\mathrm{Xtend}^{\mathbb{R}}$, respectivamente), fato que criará uma limitação em relação ao uso isolado destes herbicidas visando ao controle de soja voluntária no algodoeiro.

Especificamente no caso da tecnologia Enlist ${ }^{\circledR}$, o leque de opções de manejo pode ser ainda mais reduzido, uma vez que as cultivares apresentaram tolerância ao glyphosate e glufosinate, além do 2,4-D (GARCIA et al., 2020). Na busca por alternativas para o controle em pósemergência de plantas voluntárias de soja contendo esta nova tecnologia, Zobiole e Kalsing (2017) indicam possibilidade de uso do herbicida halauxifen-methyl. O 
G. O. TAKAHASHI et al.

halauxifen-methyl deverá ser posicionado para o controle de plantas voluntárias de soja no algodoeiro em aplicações realizadas na dessecação de pré-semeadura, uma vez que não é recomendado o uso deste herbicida em pósemergência da cultura (ZIMMER; YOUNG; JOHNSON, 2018).

Os dados referentes a avaliação de 21 DAA, indicam que o trifloxysulfuron isolado, e as associações de pyrithiobac ou trifloxysulfuron com glufosinate, proporcionaram elevados níveis de controle sobre as plantas voluntárias de soja ( $\geq 96,0 \%$ ), assemelhando ao desempenho obtido com a aplicação do dicamba isolado, bem como das associações entre 2,4-D + glufosinate e dicamba + glufosinate (Tabela 4). Pyrithiobac e trifloxysulfuron são os únicos herbicidas com espectro de controle sobre plantas daninhas dicotiledôneas registrados para uso em pós-emergência do algodoeiro em cultivares convencionais, constituindo-se como ferramentais fundamentais para o manejo da comunidade infestante desta cultura (ARANTES et al., 2014). Além disso, Braz et al. (2013a) já indicaram a possibilidade de uso destes herbicidas para o controle de soja $\mathrm{RR}^{\circledR}$, demonstrando que a eficácia dependente da dose utilizada, bem como estádio das plantas voluntárias no momento da aplicação.

$\mathrm{Na}$ última avaliação realizada aos 28 DAA, excluindo-se os herbicidas glyphosate, S-metolachlor e glufosinate aplicados de forma isolada, todos os demais tratamentos proporcionaram níveis de controle de soja voluntária superiores a 70,0\% (Tabela 4). Contudo, os tratamentos que apresentaram maior eficácia no controle químico destas plantas voluntárias, constituíram-se da aplicação isolada de dicamba, além das associações de glufosinate com 2,4-D, dicamba, pyrithiobac e trifloxysulfuron. Pela dificuldade de controle de soja em estádios avançados de desenvolvimento, bem como o elevado potencial de interferência que estas plantas voluntárias apresentam sobre o algodoeiro, deve-se sempre preconizar o uso de tratamentos que proporcionem eficácia acima de $90,0 \%$.

Semelhantemente ao comportamento observado para o estudo realizado com a cultivar de soja $\mathrm{LL}^{\circledR}$, no presente experimento visualizou-se que os tratamentos com maior eficácia no controle de soja $\mathrm{RR}^{\circledR}$ voluntária também foram capazes de impor restrições na taxa de crescimento das plantas, bem como redução na massa seca de parte aérea (Tabela 5). Para se ter uma noção da redução imposta pelos herbicidas com maior eficácia no controle de soja voluntária sobre estas variáveis-respostas, visualizou-se redução de $\approx 45,9$ e $78,8 \%$ em comparação com à testemunha sem aplicação, nos valores de altura e massa seca de parte aérea, respectivamente.

Tabela 5. Altura e massa seca de parte aérea de plantas voluntárias de soja, cultivar BRS Flecha RR2 IPRO ${ }^{\circledR}$ (tolerante ao glyphosate), submetidas à aplicação de herbicidas em pós-emergência. Rio Verde (GO), 2019.

\begin{tabular}{lcccc}
\hline \multirow{2}{*}{ Herbicida $\left(\mathrm{g} \mathrm{ha}^{-1}\right)$} & \multicolumn{2}{c}{ Altura de plantas $(\mathrm{cm})$} & \multicolumn{2}{c}{ Massa seca $(\mathrm{g})$} \\
\cline { 2 - 5 } & Sem AG & Com AG & Sem AG & ${\text { Com } \mathrm{AG}^{1 /}}^{1 /}$ \\
\hline 2,4-D (644) & $26,0 \mathrm{Ab}$ & $22,0 \mathrm{Aa}$ & $2,8 \mathrm{Abcd}$ & $1,0 \mathrm{Bb}$ \\
Dicamba (600) & $23,0 \mathrm{Ab}$ & $20,5 \mathrm{Aa}$ & $1,1 \mathrm{Ad}$ & $0,6 \mathrm{Ab}$ \\
Glyphosate (480) & $37,4 \mathrm{Aa}$ & $22,8 \mathrm{Ba}$ & $4,1 \mathrm{Aab}$ & $3,2 \mathrm{Aa}$ \\
Pyrithiobac (84) & $24,4 \mathrm{Ab}$ & $23,8 \mathrm{Aa}$ & $3,1 \mathrm{Aabcd}$ & $1,1 \mathrm{Bab}$ \\
Trifloxysulfuron (3) & $24,0 \mathrm{Ab}$ & $22,5 \mathrm{Aa}$ & $1,9 \mathrm{Acd}$ & $2,5 \mathrm{Bab}$ \\
S-metolachlor (1152) & $37,5 \mathrm{Aa}$ & $20,3 \mathrm{Ba}$ & $3,9 \mathrm{Aabc}$ & $1,6 \mathrm{Bab}$ \\
Sem herbicida & $41,3 \mathrm{Aa}$ & $23,8 \mathrm{Ba}$ & $5,1 \mathrm{Aa}$ & $2,2 \mathrm{Bab}$ \\
\hline CV (\%) & \multicolumn{3}{c}{39,73} \\
\hline
\end{tabular}

${ }^{1 /}$ Com AG = associado ao glufosinate $\left(200 \mathrm{~g} \mathrm{ha}^{-1}\right)$. ${ }^{*}$ Médias seguidas por letras distintas maiúsculas na linha e minúsculas na coluna diferem entre si pelo teste de Tukey $(p \leq 0,05)$.

Apesar de alguns tratamentos não terem proporcionado níveis de controle acima de $80,0 \%$, quando se observa as reduções na altura de plantas, bem como massa seca de parte aérea, este resultado pode ser importante do ponto de vista de manejo integrado de plantas daninhas no algodoeiro. Esta afirmação pode ser subsidiada pela maior facilidade em que plantas de menor porte e/ou que estejam sob estresse químico apresentam em ser controladas com o uso de herbicidas, fato que poderia facilitar o manejo químico das plantas voluntárias de soja com aplicações sequenciais de herbicidas (BRAZ et al., 2013a). Ademais, outra possibilidade em termos de manejo integrado refere-se ao controle cultural da soja voluntária pelo sombreamento imposto pelo algodoeiro, visto que as plantas voluntárias poderão apresentar menor taxa de crescimento quando submetidas ao estresse provocado pelos herbicidas (BRAZ et al., 2013b).

Com base nos resultados visualizados nos experimentos, fica evidente que visando à manutenção do sistema produtivo do algodoeiro em segunda safra sustentável, a escolha do tipo de tecnologia contida tanto na cultivar de soja explorada como cultura antecessora, como o material de algodoeiro a ser semeada consistem em passos de fundamental importância. A partir da análise das tecnologias que conferem tolerância a herbicidas empregadas tanto na soja como no algodoeiro, será possível definir qual a estratégia mais adequada para o controle químico das plantas voluntárias de soja na cultura do algodão. 
G. O. TAKAHASHI et al.

\section{Conclusões}

Há tolerância diferencial entre os cultivares de soja contendo diferentes transgenias quanto a sensibilidade aos herbicidas utilizados para o controle de plantas voluntárias.

Glyphosate e dicamba, independentemente da associação ou não com glufosinate, e 2,4-D + glufosinate apresenta eficácia para o controle de soja tolerante ao glufosinate $\mathrm{LL}^{\circledR}$.

Para a cultivar de soja tolerante ao glyphosate $\mathrm{RR}^{\circledR}$, a aplicação isolada de dicamba, 2,4-D e trifloxysulfuron proporciona controle eficaz das plantas voluntárias. Para esta cultivar, o glufosinate aplicado isoladamente não apresenta eficácia, entretanto, quando em associações com dicamba, 2,4-D, pyrithiobac, trifloxysulfuron ou Smetolachlor, há controle eficaz das plantas voluntárias de soja.

\section{Referências}

Arantes, J. G. Z.: Constantin, J.; Oliveira Jr. R. S.; Braz, G. B. P.; Barbosa, C. A. S.; Brugnera, P.; Gemelli, A. Selectivity of chemical weed control systems in conventional cotton. Planta Daninha, 32(4): 827-841, 2014.

Bond, J. A.; Walker, T. W. Control of volunteer glyphosateresistant soybean in rice. Weed Technology, 23( 2): 225230, 2009 .

Botha, G. M.; Burgos, N. R.; Gbur, E. E.; Alcober, E. A.; Salas, R. A.; Scott, R. C. Interaction of glufosinate with 2,4$\mathrm{D}$, dicamba, and tembotrione on glyphosate-resistant Amaranthus palmeri. Journal of Experimental Agriculture International, 4(4): 427-442, 2014.

Braz, G. B. P.; Oliveira Jr, R. S.; Constantin, J.; Oliveira Neto, A. M.; Dan, H. A.; Guerra, N.; Takano, H. K. Alternativas para o controle de soja $\mathrm{RR}^{\circledR}$ voluntária na cultura do algodoeiro. Bioscience Journal, 29(2): 360-369, 2013a.

Braz, G. B. P.; Oliveira Jr, R. S.; Constantin, J.; Takano, H. K.; Gheno, E. A.; Biffe, D. F. Atividade residual de pyrithiobac-sodium aplicado ao solo, visando ao controle de plantas voluntárias de soja $R^{\circledR}$. Global Science and Technology, 6(3): 99-107, 2013b.

Cardoso, G. D.; Alves, P. L. D. C. A.; Beltrão, N. E. D. M.; Vale, L.S. D. Períodos de interferência das plantas daninhas em algodoeiro de fibra colorida 'BRS Safira'. Revista Ciência Agronômica, 41(3): 456-462, 2010.

CONAB - Companhia Nacional de Abastecimento. (2020). Acompanhamento da safra brasileira de grãos. 104 p. v. 7 safra 2019/2020. Companhia Nacional de Abastecimento, Disponível em: <https://www.conab.gov.br/info-agro/safras/ graos/boletim-da-safra-de-graos>. Acesso em: 03 fev 2020).
Craigmyle, B. D.; Ellis, J. M.; Bradley, K. W. Influence of weed height and glufosinate plus 2,4-D combinations on weed control in soybean with resistance to $2,4-D$. Weed Technology, 27(2): 271-280, 2013.

EMBRAPA - Empresa Brasileira de Pesquisa Agropecuária. Sistema brasileiro de classificação de solos. Rio de Janeiro, RJ: EMBRAPA Solos, Centro Nacional de Pesquisa de Solos, 2018.

Ferreira, A. D. B.; Borin, A. L. D. C.; Brito, G. G.; Silva Filho, J. L.; Bogiani, J. C. Épocas de semeadura, cultivares e densidades de plantas para algodão adensado em segunda safra. Pesquisa Agropecuária Tropical, 25(4): 397-405, 2015 .

Ferreira, D. F. Sisvar: a computer statistical analysis system. Ciência e Agrotecnologia, 35(6): 1039-1042, 2011.

Garcia, J. R.; Vargas, A. A. M.; Perboni, L. T.; Souza, E. A.; Tessaro, D.; Lucio, F. R.; Agostinetto, D. Physiological attributes of Enlist E3 $3^{\mathrm{TM}}$ soybean seed submitted to herbicides application. Planta Daninha, 38: e020220418, 2020.

Green, J. M. Evolution of glyphosate-resistant crop technology. Weed Science, 57(1): 108-117, 2009.

Lee, D. R.; Miller, D. K.; Blouin, D. C.; Clewis, S. B.; Everman, W. J. Glyphosate-resistant soybean interference in glyphosate-resistant cotton. Journal of Cotton Science, 13 (2): 178-182, 2009.

Lima, D. B. C.; Silva, A. G.; Procópio, S. O.; Barroso, A. L. L.; Dan, H. A. Controle químico de plantas voluntárias de soja Roundup Ready ${ }^{\circledR}$ em diferentes estádios de desenvolvimento. Revista Caatinga, 24(3): 64-70, 2011.

Oliveira Neto, A. M.; Constantin, J.; Oliveira Jr, R. S.; Barroso, A. L. L.; Braz, G. B. P.; Guerra, N.; Gheno, E. A. Selectivity of fomesafen to cotton. Planta Daninha, 33(4): 759-770, 2015.

Raimondi, M. A.; Oliveira Jr, R. S.; Constantin, J.; Franchini, L. H. M.; Blainski, É.; Raimondi, R. T. Weed interference in cotton plants grown with reduced spacing in the second harvest season. Revista Caatinga, 30(1): 1-12, 2017.

SBCPD - Sociedade Brasileira da Ciência das Plantas Daninhas. Procedimentos para instalação, avaliação e análise de experimentos com herbicidas. Londrina: $42 \mathrm{p}$. 1995.

Toledo, A. D.; Tabile, R. A.; Silva, R. P. D.; Furlani, C. E.; Magalhães, S. C.; Costa, B. O. Caracterização das perdas e distribuição de cobertura vegetal em colheita mecanizada de soja. Engenharia Agrícola, 28(4): 710-719, 2008. 
G. O. TAKAHASHI et al.

USDA. (2021). World Agricultural Production. 40 p. v. 3. Março de 2021. Disponível em: https://apps.fas.usda.gov/ psdonline/circulars/production.pdf. Acesso em: 09 mar. 2021.

Zimmer, M.; Young, B. G.; Johnson, W. G. Weed control with halauxifen-methyl applied alone and in mixtures with 2,4-D, dicamba, and glyphosate. Weed Technology, 32(5): 597-602, 2018.

Zobiole, L. H. S.; Kalsing, A. Eficácia de misturas formuladas contendo halauxifen-methyl no controle de soja voluntária DAS-444Ø6-6 (Enlist E3TM). Revista Brasileira de Herbicidas, 16(3):192-197, 2017. 